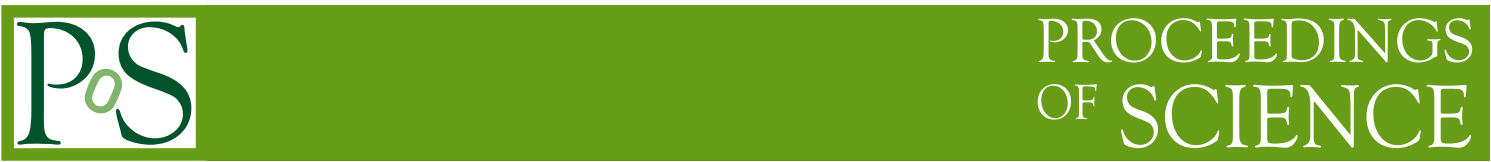

\title{
Few-Body Lattice Calculations
}

\author{
Paulo F. Bedaque* \\ University of Maryland \\ E-mail: bedaque@umd.edu
}

We review the recent advances in the calculation of the forces between hadron using lattice QCD. Particular attention is payed to the case of nuclear forces. The issues facing this project and some of the ideas put forward to solve them are briefly discussed.

8th Conference Quark Confinement and the Hadron Spectrum

September 1-6 2008

Mainz, Germany

* Speaker. 


\section{Introduction}

The nuclear force is the basic building block of nuclear physics. Since the discovery of the strong interactions a great deal was learned about it experimentally and phenomenological models and low energy effective theories were devised to describe it. Still, the advent of QCD almost 40 years ago has had little impact on our understanding of nuclear forces. This is a fate shared by many other low energy features of the strong interactions which also remain essentially uncalculable from first principles.

Lattice QCD is a mature technique, almost as old as QCD itself, designed to address this general class of problems. The constant influx of new ideas in the field and the continuing development of computer hardware has finally allowed lattice QCD in the last few years to have a real impact on important phenomenological questions. Up to recently, however, lattice QCD had been mostly used in the calculation of simple observables like hadron masses and decay constants. The time now seems ripe for the use of lattice QCD on more challenging problems, the origins of the nuclear force being naturally at the top of the list. In this review we discuss some of the main ideas involved in this programme and the first results to come out of it.

Besides the purely theoretical reasons to be interested in a first principles calculation of the nuclear force (and other related processes), there are also several more "practical" motivations. Among them we cite:

- A nuclear force calculation is a necessary stepping stone towards the calculation of other twohadron observables that impact, at a phenomenological relevant level, electroweak processes used, for instance, in neutrino physics.

- The interaction between nucleons and hyperon is poorly known experimentally and very relevant in determining the fate of strangeness in neutron stars.

- Even though nucleon-nucleon phase shifts are known experimentally with good precision, their dependence on the fundamental constants (quark masses, ...), valuable in studies of variation of fundamental constants over cosmological times, is unknown.

- The nuclear three-body force is poorly know phenomenologically and very important to nuclear structure calculations.

- The study of meson interaction on the lattice provides another window into the value of the low energy constants that define the chiral dynamics regime.

\section{Lüscher method}

Lattice field theory can only be done in imaginary time, that is, in euclidean as opposed to Minkowski space ${ }^{1}$. A natural question that is posed is how to relate matrix elements computes in euclidean space to real, Minkowski space physical observables. For some observables, like particle masses, there is a well know recipe of how to do that. Let us take the case of the positive pion

\footnotetext{
${ }^{1}$ The periodic attempts at using stochastic quantization in order to work on Minkowski space, promising as may be in other areas, are unlike to have an impact on the calculation of scattering amplitudes
} 
mass. One can compute the imaginaty time correlator $C(t)=\left\langle\pi^{-}\left(\mathbf{r}^{\prime}, \mathbf{t}\right) \pi^{+}(\mathbf{r}, \mathbf{0})\right\rangle$, with $\pi^{+}$being any operator made up of quarks and gluons with the quantum numbers of the pion like, for instance, $\bar{q} \gamma_{5} \tau^{+} q$. Using standard quantum mechanics arguments we can arrive at

$$
\begin{aligned}
C(t) & =\sum_{n}\left\langle 0\left|e^{H t} \pi^{-}\left(\mathbf{r}^{\prime}, 0\right) e^{-H t}\right| n\right\rangle\left\langle n\left|\pi^{+}(\mathbf{r}, 0)\right| 0\right\rangle \\
& =\sum_{n} e^{-E_{n} t}\left\langle 0\left|\pi^{-}\left(\mathbf{r}^{\prime}, 0\right)\right| n\right\rangle\left\langle n\left|\pi^{+}(\mathbf{r}, 0)\right| 0\right\rangle \\
& \rightarrow e^{-m_{\pi} t} \mid\left.\left\langle\text { pion at rest }\left|\pi^{+}(\mathbf{r}, 0)\right| 0\right\rangle\right|^{2},
\end{aligned}
$$

where $|n\rangle$ is the n-th eigenstate of the hamiltonian $H$ with energy $E_{n}$. At large times $t$ the sum is dominated by the energy with the smallest energy among those with the quantum numbers of one positive pion (namely, the positive pion at rest). Thus, by measuring the long time behavior of $C(t)$ we can extract the pion mass. It is not so easy to extract scattering information on the lattice. The naive attempt using the two pion correlator leads to

$$
C_{2}(t)=\left\langle\pi^{-}\left(\mathbf{r}^{\prime}, \mathbf{t}\right) \pi^{-}\left(\mathbf{r}^{\prime}, \mathbf{t}\right) \pi^{+}(\mathbf{r}, \mathbf{0}) \pi^{+}(\mathbf{r}, \mathbf{0})\right\rangle \rightarrow \mathbf{e}^{-2 \mathbf{m}_{\pi} \mathbf{t}} \mid\left.\left\langle 2 \text { pions at rest }\left|\pi^{+}(\mathbf{r}, 0) \pi^{+}(\mathbf{r}, 0)\right| 0\right\rangle\right|^{2},
$$

which does not bring any information about the pion interactions. At infinite volume we find then that the euclidean correlators bring no information about the scattering properties [1]. At finite volume however, the two particles cannot be well separated and the energy of the ground state of two particles will include the interaction energy [2]

$C_{2}(t)=\left\langle\pi^{-}\left(\mathbf{r}^{\prime}, t\right) \pi^{-}\left(\mathbf{r}^{\prime}, t\right) \pi^{+}(\mathbf{r}, 0) \pi^{+}(\mathbf{r}, 0)\right\rangle \rightarrow e^{-\left(2 m_{\pi}+\Delta E\right) t} \mid\left.\left\langle 2\right.$ pions at rest $\left.\left|\pi^{+}(\mathbf{r}, 0) \pi^{+}(\mathbf{r}, 0)\right| 0\right\rangle\right|^{2}$.

The shift in the two-particle energy levels is due to the interactions so teir measurement should teach us something about it. In fact, in the case of small interaction we can use first order perturbation theory around the free two-particle, zero momentum wavefunction $\Psi\left(\mathbf{r}, \mathbf{r}^{\prime}\right)=1 / L^{3}$ ( $L$ is the size of the box), the shift in energy due to the interaction between the particles is

$$
\Delta E=\int d^{3} \mathbf{r} d^{3} \mathbf{r}^{\prime} \Psi^{*}\left(\mathbf{r}, \mathbf{r}^{\prime}\right) V\left(\mathbf{r}-\mathbf{r}^{\prime}\right) \Psi\left(\mathbf{r}, \mathbf{r}^{\prime}\right)=\frac{1}{L^{3}} \int d^{3} \mathbf{r} V(\mathbf{r})=\frac{4 \pi a}{M L^{3}},
$$

where $a$ is the scattering length between the particles given, in the Born approximation, by $a=$ $M / 4 \pi \int d^{3} \mathbf{r} V(\mathbf{r})$.

The relation between phase shifts and finite volume two-particle energy shifts is given, in full generality by the Luscher formula [3]

with

$$
\sqrt{M \Delta E} L \cot \delta(\Delta E)=\mathscr{S}\left(\frac{M \Delta E L^{2}}{4 \pi^{2}}\right),
$$

$$
\mathscr{S}\left(\frac{M \Delta E L^{2}}{4 \pi^{2}}\right)=\lim _{\Lambda \rightarrow \infty} \sum_{|\mathbf{n}|<\Lambda} \frac{1}{\mathbf{n}^{2}-\frac{M \Delta E L^{2}}{4 \pi^{2}}}-4 \pi \Lambda .
$$

. If one measures the energy levels of two particles in a box with enough precision this formula can be used to learn about the phase shift at that particular value of the energy. By changing the 
value of the box size $L$ the phase shift at other energy values can be probed ${ }^{2}$. In the case where the scattering length $a$ is much smaller than the lattice size $L$, the perturbative result is recovered

$$
\Delta E=\frac{4 \pi a}{M L^{3}}\left(1+c_{1} \frac{a}{L}+c_{2} \frac{a^{2}}{L^{2}}+\cdots\right)
$$

where $c_{1}, c_{2}$ are known numerical coefficients. The leading term of the formula above can be obtained by a simple use of perturbation theory. It is important to stress however, that the finite energy phase shifts can be obtained even if $a \gg L$. In other words, we can learn about the deuteron using boxes much smaller than the deuteron itself. This is particularly important in nuclear physics as the scattering length in the spin singlet case is very large (of the order of $20 \mathrm{fm}$ ) [5]. The condition on the size of the box for the Luscher formula to be valid is that the range of the interaction (in the nuclear case of order $1 / m_{\pi}$ ) should be much smaller than $L$. That is a much weaker condition than $a \ll L$ since, in the nuclear case $a \gg 1 / m_{\pi}$.

\section{Current results}

Besides some pioneer work in the past in the quenched approximation [6] and on the potential between heavy mesons [?], only recently the study of hadron interaction in the lattice picked up steam. The Nuclear Physics with Lattice QCD (NPLQCD) collaboration has pursued a vigorous program in this direction by calculating several hadronic scattering observables using the method outlined above. Before discussing the results let me discuss a few details of these simulations. All the calculations discussed here were done using gauge field configurations generated by the MILC collaboration [7] (for different purposes). The are fully dynamical (meaning, the fermion determinant is included in the probability distribution). It uses the asqtad action [8] which is improved so the largest discretization errors are of order $\alpha_{s} a^{2}$ ( $a$ is the lattice spacing and $\alpha_{s}$ the strong coupling constant). The quark masses for the up and down quarks are chosen so the pion has masses $m_{\pi}=294,348$ and $484 \mathrm{MeV}$. NPLQCD computed quark propagators for the valence sector moving in these gauge field configurations using a different discretization (domain-wall quarks) of the quark action. Domain-wall quarks were used because, despite being numerically more expensive, they have an almost exact chiral symmetry. In particular, the leading source of discretization errors (of order $a$ ), which breaks chiral symmetry, is automatically absent of this formulation. The lattice spacing of these lattices is about 0.125 (extracted from the Sommer scale) fm and the total size of the lattice is about $2.5 \mathrm{fm}$.

\section{1 $I=2 \pi \pi$ scattering length}

From the point of view of the lattice, the simplest hadronic interaction to be studied is the isospin $I=2$ channel of the $\pi \pi$ system. The value of the scattering length extracted at three different pion masses can be extrapolated to the realistic pion masses using the chiral perturbation theory one-loop result

\footnotetext{
${ }^{2}$ Another, and cheaper, way of measuring the phase shifts at other values of the energy is to change the boundary conditions [4].
} 


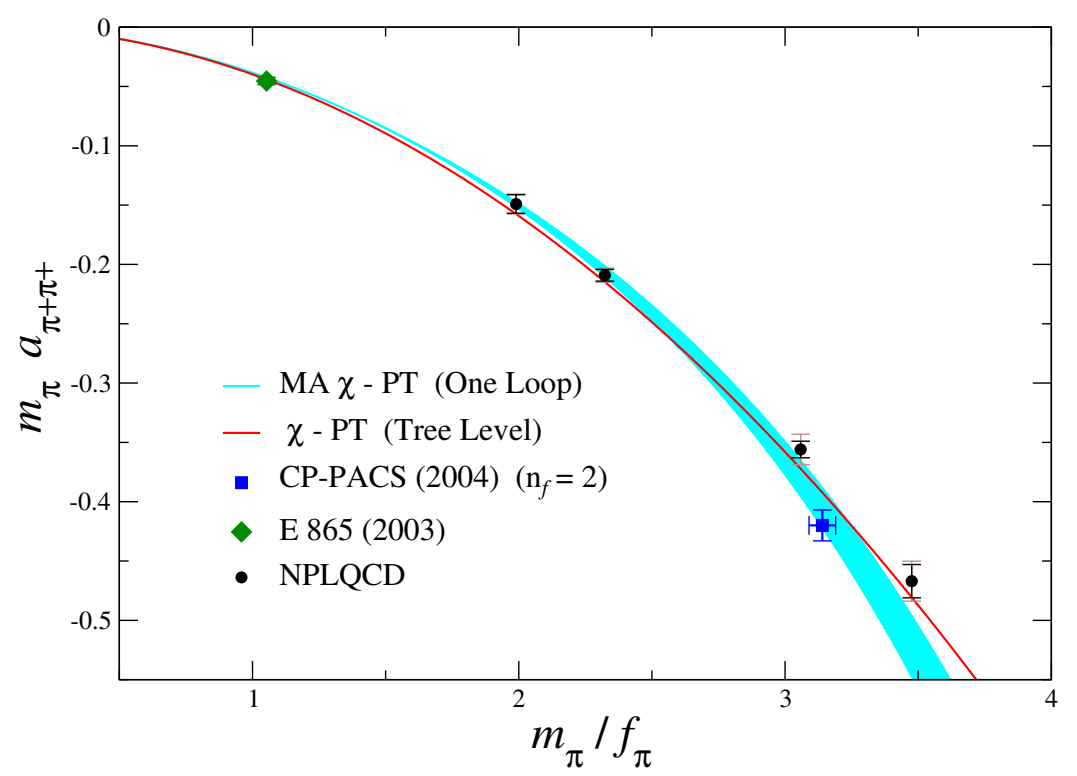

Figure 1: Scattering length (in units of $m_{\pi}^{-1}$ ) as a function of pion mass (in units of $f_{\pi}$ ). Black dots represent the NPLQCD calculations with statistical (dark bars) and systematic (light bars) uncertainties. Also shown are the experimental value from [10] (diamond) and the lowest quark mass result of the $N_{F}=2$ dynamical calculation of CP-PACS [11] (square). The blue band corresponds to a weighted fit to the lightest three data points using the one-loop mixed-action $\chi$ PT result and the red line to the tree level $\chi$ PT result. The experimental data is not used in the fits. Figure from [13]

$$
m_{\pi} a=-\frac{m_{\pi}^{2}}{8 \pi f_{\pi}^{2}}\left[1+\frac{3 m_{\pi}^{2}}{16 \pi f_{\pi}^{2}}\left(\log \left(\frac{m_{\pi}^{2}}{\mu^{2}}+l_{\pi \pi}(\mu)\right)\right)\right],
$$

where $l_{\pi \pi}(\mu)=8\left(l_{1}+l_{2}\right)+2\left(l_{3}-l_{4}\right)$ is a combination of Gasser-Leutwyler coefficients parameterizing some short-distance effects and $\mu$ is the renormalization scale. Since $f_{\pi}$ is also calculated using the same pion masses the formula above can be used to perform a one parameter $\left(l_{\pi \pi}\right)$ fit to the numerical results. The initial NPLQCD result [12] was superseded by the one in [13]. The results are summarized on Figure (1). The result is consistent to sevral other theoretical and experimental determinations (see [16] for an overview).

Several of the systematic errors in this calculation were estimated using $\chi$ PT techniques. The discretization errors are of order $a^{2} \Lambda_{Q C D} \approx 1 \%$ [14]. The finite volume effects (exponentially suppressed and not included in the Luscher formula) can be estimated by chiral perturbation theory and are also of the order of a few percent [15]. It is worthwhile emphasizing the ingredients that allow for such a precise calculation of a scattering observable at the physical value of the pion mass. They are: i) relatively large signal to noise ratio, ii) the existence of an exact result ( $m_{\pi} a=0$ for $m_{\pi}=0$ ) anchoring the extrapolation down to physical quark masses and iii) a reliable extrapolation formula standing on solid grounds coming from chiral perturbation theory. Some of these desirable features are unfortunatelly not present in other scattering observables.

Scattering lengths for $\pi^{+} K^{+}$[17] and $K^{+} K^{+}$[18] were also calculated with similar level of precision. Also, multi-meson systems were studied with similar techniques [19]. 


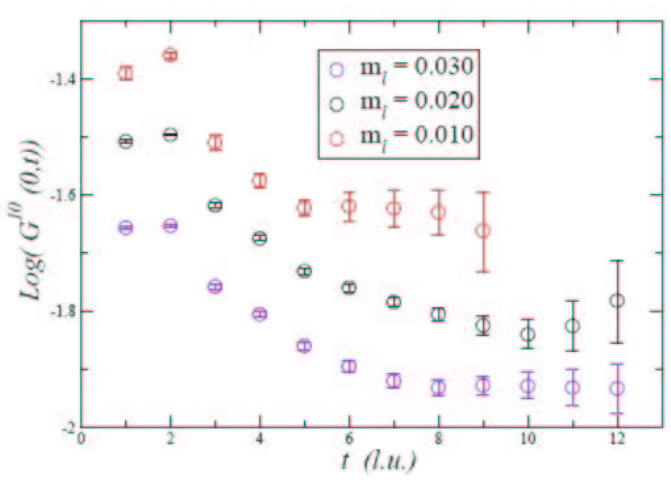

Figure 2: Log of the correlator ratio for the spin singlet nucleon-nucleon channel at three different quark masses.

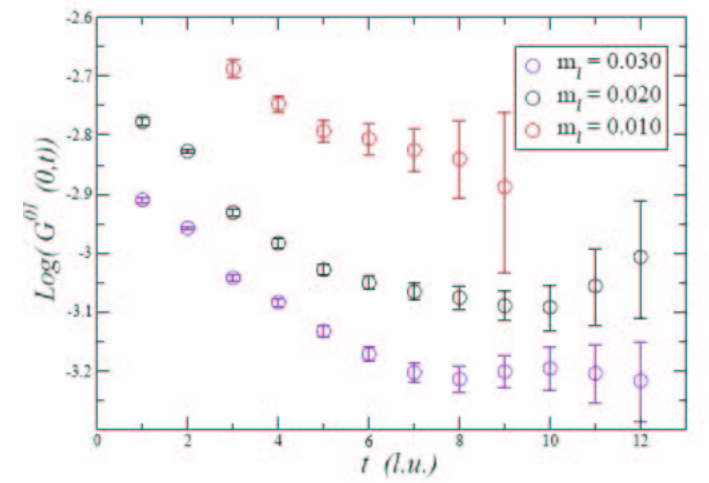

Figure 3: $\log$ of the correlator ratio for the spin triplet nucleon-nucleon channel at three different quark masses.

\subsection{Nucleon-nucleon scattering length}

At low energies nucleons are non-relativistic and it is useful to describe their interaction through a non-relativistic potential. It should be kept in mind, however, that the non-relativistic potential is a construct belonging to the low-energy effective theory of QCD describing low energy nuclear phenomena and cannot be uniquely defined in QCD. On physical grounds, we don't expect that short distance (high momentum) interaction between two nucleons to be describable by a potential so the nuclear potential at short distances (say, smaller than $0.5 \mathrm{fm}$ ) is not well defined. For this reason the study of the nuclear forces from QCD does not pass through the derivation of the nuclear potential (including its short distance piece) but proceeds directly to the calculation of phase shifts. Of course, many potentials, with different short-distance behaviors can be concocted to reproduce the low energy data (experimental or from numerical QCD), as it has been done phenomenologically with great accuracy. But since the short-distance behavior is somewhat arbitrary (in the language of effective field theory, it is "regulator dependent") it cannot be deduced from QCD. This is in contrast to the interaction between two infinitely heavy particles where an adiabatic potential is well defined at all distances. Any attempt at a lattice QCD calculation of the nuclear potential will be plagued, at short distances, by the arbitrarity in the choice of interpolating fields for the nucleons. The phase shifts, as we argued above, are related to energy levels and are independent of this choice.

On figures 2 and 3 [20] we show the results for the correlator ratio

$$
\frac{\left\langle N(t) N(t) N^{\dagger}(0) N^{\dagger}(0)\right\rangle}{\left\langle N(t) N^{\dagger}(0)\right\rangle^{2}} \stackrel{t \rightarrow \infty}{\rightarrow} e^{-\Delta E t}
$$

whose long time behavior directly measures the energy shift $\Delta E$, for the two spin channels (singlet and triplet) in the nucleon-nucleon system. Compared to the $\pi \pi$ case one outstanding feature is the decreasing signal-to-noise ratio for large $t$. This can be understood from simple arguments that indicate that

$$
\text { signal to noise ratio } \sim e^{-\left(2 M-3 m_{\pi}\right) t},
$$




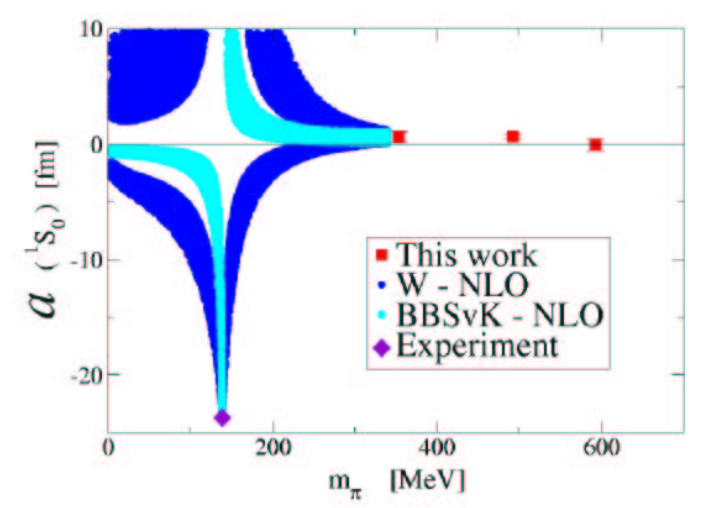

Figure 4: Interpolation/extrapolation between the lattice QCD and the experimental results in the spin singlet channel.

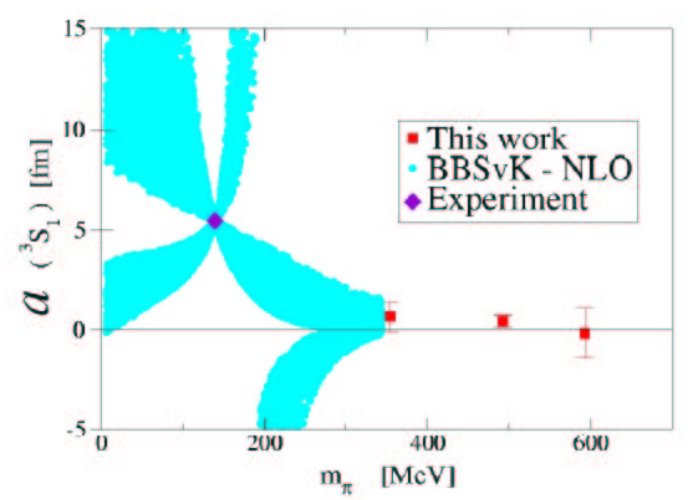

Figure 5: Interpolation/extrapolation between the lattice QCD and the experimental results in the spin triplet channel.

where $M$ is the nucleon mass. Consequently, the uncertainty in the extraction of the scattering length is much larger. Nucleon-nucleon scattering lengths however, are very large, at least for physical pion masses. Thus even upper bounds on the value of the scattering lengths contain nontrivial information. The values found are shown in table 1.

Table 1: Scattering lengths for the two nucleon-nucleon spin channels. The numbers in parentheses indicate the time range used in the fit.

\begin{tabular}{|c|c|c|}
\hline$m_{\pi}(\mathrm{MeV})$ & $a\left({ }^{1} S_{0}\right)(\mathrm{fm})$ & $a\left({ }^{3} S_{1}\right)(\mathrm{fm})$ \\
\hline $353.7 \pm 2.1$ & $0.63 \pm 0.50(5-10)$ & $0.63 \pm 0.74(5-9)$ \\
$492.5 \pm 1.1$ & $0.65 \pm 0.18(6-9)$ & $0.41 \pm 0.28(6-9)$ \\
$593.0 \pm 1.6$ & $0.0 \pm 0.5(7-12)$ & $-0.2 \pm 1.3(7-12)$ \\
\hline
\end{tabular}

The effective theory describing nucleon-nucleon scattering contains, at next-to-leading order, two undetermined constants [21]. One $\left(C_{0}\right)$ is the leading short-distance quark mass independent interaction. The other $\left(D_{2}\right)$ describes the leading short-distance quark mass dependence. Unfortunately, the regime of applicability of the nuclear effective theory is smaller than the chiral perturbation theory in the meson sector and only the lowest value of the pion mass falls (at the edge) of this range. With only one data point both $C_{0}$ and $D_{2}$ cannot be determined and a prediction for the scattering length at the physical point is not possible. However, we can use the measured value of the scattering length at the physical point and predict what the scattering length would be at different values of the quark mass. The result of these calculations are shown in figures 2 and 3. The shaded area correspond to results obtained with values of $C_{0}$ and $D_{2}$ fitting both the lowest pion mass point and the experimental datum and also conforming with the expectations of naive dimensional analysis. Two power counting schemes were used, the one advocated by Weinberg [22] and the one discussed in reference [23]. Their predictions are not significantly different and their difference suggests what the errors in this extrapolation is. 


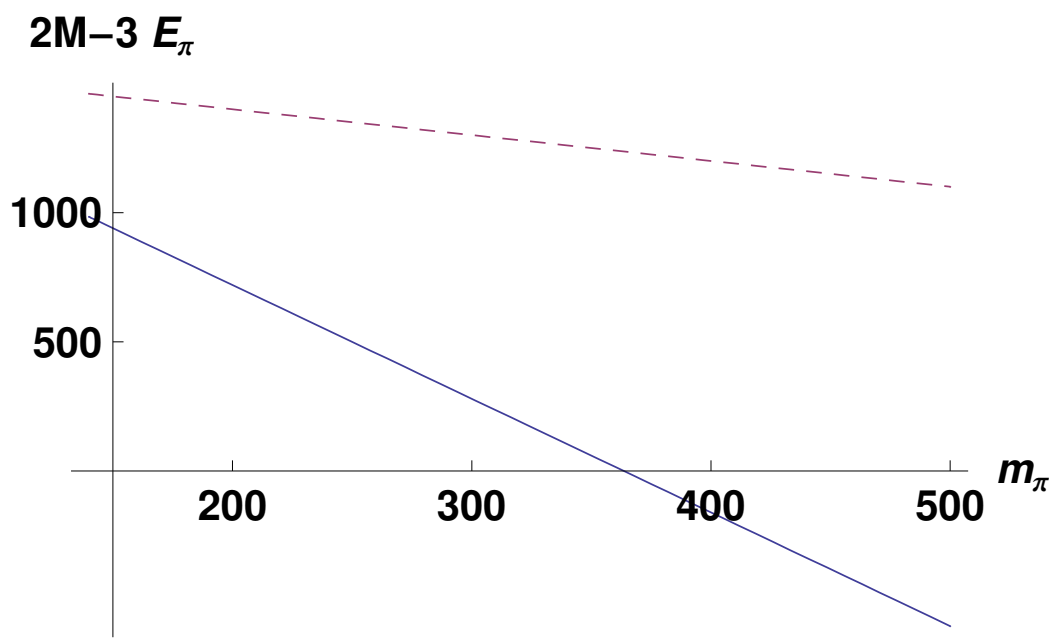

Figure 6: Estimated energy spliting controlling the signal-to-noise ratio $\left(2 M-3 E_{\pi}\right)$ in two-baryon correlators. The red dashed line is for periodic boundary conditions while the blue solid line is for one verion of the orbifold boundary condition, in which we have fixed $m_{\pi} L=4$. From reference [27].

The systematic errors in this calculation were not fully explored. The error arising from the formally exponentially suppressed corrections to the Luscher formula, were estimated in [25] and, for the pion masses used, are smaller than the statistical errors. In this reference it is pointed out that the most naive finite volume correction - the fact that the two nucleons can interact in a direct way and also through pions exchanged "around the lattice" - in fact vanish at leading order in perturbation theory.

\section{Future prospects}

It is evident that, while the study of meson interaction on the lattice is now competitive with the experiments, the study of baryon-baryon interactions is still in its infancy. Clearly, the main obstacle is the exponential growth of the statistical noise in multi-baryon correlators. Three main ideas are being pursued to address this issue. The first is the construction of better sources for the two-baryon states, on the molds of [26]. The second is the use of anisotropic lattices, with he lattice spacing in the time direction several times smaller than on the space direction. The hope is that the availability of extra time slices will allow for a determination of the energy levels before the signal is degraded by statistical noise. Finally, a recent idea to virtually eliminate the problem has been put forward recently but that remains still untested [27]. Suppose that, somehow, the zero mode of the pion states is eliminated from the lattice. Equation (4.1) is changed to

$$
\text { signal to noise ratio } \sim e^{-\left(2 M-3 E_{\pi}\right) t},
$$

where $E_{\pi}$ is the minimum energy of the pion. If the lowest pion energy is high enough, the exponential increase o the statistical noise can be alleviated or entirely suppressed. The zero-mode of the pion can be easily eliminated by imposing different boundary conditions on the pion filed like, or instance, $\pi(0)=0$. The problem is that only the boundary conditions satisfied by quarks and 
gluons can be controlled in a simulation. The solution advocated in [27] is to double the lattice size in one direction but keep the quark and gluon fields in the extra half constrained to be the parity reversal of the fields in the other half. In other words, one performs the calculation on a parity orbifolded spacetime, in a manner similar to that used in extra-dimension theories of grand unified theories [28]. As a consequence, the pion filds will satisfy $\pi(-z)=-\pi(z)$ and the zero mode is eliminated. The change in the lattice action implementing this construction is only the addition of a boundary term along the $z=0$ plane. Its precise form (dependent on the particular fermion action used) is entirely determined by the orbifold condition. The potential improvement on the rate of decrease in the signal-to-noise ratio is illustrated on Figure (6).

\section{References}

[1] L. Maiani and M. Testa, Phys. Lett. B 245 (1990) 585.

[2] H. W. Hamber, E. Marinari, G. Parisi and C. Rebbi, Nucl. Phys. B 225, 475 (1983).

[3] M. Luscher, Nucl. Phys. B 354, 531 (1991); M. Luscher, Commun. Math. Phys. 105 (1986) 153.

[4] P. F. Bedaque, Phys. Lett. B 593, 82 (2004) [arXiv:nucl-th/0402051]; C. T. Sachrajda and G. Villadoro, Phys. Lett. B 609, 73 (2005) [arXiv:hep-lat/0411033]. P. F. Bedaque and J. W. Chen, Phys. Lett. B 616, 208 (2005) [arXiv:hep-lat/0412023]; G. M. de Divitiis, R. Petronzio and N. Tantalo, Phys. Lett. B 595, 408 (2004) [arXiv:hep-lat/0405002]; J. M. Flynn, A. Juttner and C. T. Sachrajda [UKQCD Collaboration], Phys. Lett. B 632, 313 (2006) [arXiv:hep-lat/0506016].

[5] S. R. Beane, P. F. Bedaque, A. Parreno and M. J. Savage, Phys. Lett. B 585, 106 (2004) [arXiv:hep-lat/0312004].

[6] M. Fukugita, Y. Kuramashi, M. Okawa, H. Mino and A. Ukawa, Phys. Rev. D 52, 3003 (1995) [arXiv:hep-lat/9501024].

[7] C. W. Bernard et al., Phys. Rev. D 64, 054506 (2001) [arXiv:hep-lat/0104002].

[8] K. Orginos and D. Toussaint [MILC collaboration], Phys. Rev. D 59, 014501 (1999) [arXiv:hep-lat/9805009];

[9] K. Orginos, D. Toussaint and R. L. Sugar [MILC Collaboration], Phys. Rev. D 60, 054503 (1999) [arXiv:hep-lat/9903032].

[10] S. Pislak et al., Phys. Rev. D 67, 072004 (2003) [arXiv:hep-ex/0301040].

[11] T. Yamazaki et al. [CP-PACS Collaboration], Phys. Rev. D 70, 074513 (2004) [arXiv:hep-lat/0402025].

[12] S. R. Beane, P. F. Bedaque, K. Orginos and M. J. Savage [NPLQCD Collaboration], Phys. Rev. D 73, 054503 (2006) [arXiv:hep-lat/0506013].

[13] S. R. Beane, T. C. Luu, K. Orginos, A. Parreno, M. J. Savage, A. Torok and A. Walker-Loud, Phys. Rev. D 77, 014505 (2008) [arXiv:0706.3026 [hep-lat]].

[14] J. W. Chen, D. O'Connell and A. Walker-Loud, Phys. Rev. D 75, 054501 (2007) [arXiv:hep-lat/0611003].

[15] P. F. Bedaque, I. Sato and A. Walker-Loud, Phys. Rev. D 73, 074501 (2006) [arXiv:hep-lat/0601033].

[16] H. Leutwyler, arXiv:hep-ph/0612112. 
[17] S. R. Beane, P. F. Bedaque, T. C. Luu, K. Orginos, E. Pallante, A. Parreno and M. J. Savage, Phys. Rev. D 74, 114503 (2006) [arXiv:hep-lat/0607036].

[18] S. R. Beane, T. C. Luu, K. Orginos, A. Parreno, M. J. Savage, A. Torok and A. Walker-Loud [NPLQCD Collaboration], Phys. Rev. D 77, 094507 (2008) [arXiv:0709.1169 [hep-lat]].

[19] S. R. Beane, W. Detmold, T. C. Luu, K. Orginos, M. J. Savage and A. Torok, Phys. Rev. Lett. 100, 082004 (2008) [arXiv:0710.1827 [hep-lat]]; W. Detmold, M. J. Savage, A. Torok, S. R. Beane, T. C. Luu, K. Orginos and A. Parreno, Phys. Rev. D 78, 014507 (2008) [arXiv:0803.2728 [hep-lat]]; W. Detmold, K. Orginos, M. J. Savage and A. Walker-Loud, Phys. Rev. D 78, 054514 (2008) [arXiv:0807.1856 [hep-lat]].

[20] S. R. Beane, P. F. Bedaque, K. Orginos and M. J. Savage, Phys. Rev. Lett. 97, 012001 (2006) [arXiv:hep-lat/0602010].

[21] P. F. Bedaque and U. van Kolck, Ann. Rev. Nucl. Part. Sci. 52, 339 (2002) [arXiv:nucl-th/0203055].

[22] S. Weinberg, Nucl. Phys. B 363, 3 (1991).

[23] S. R. Beane, P. F. Bedaque, M. J. Savage and U. van Kolck, Nucl. Phys. A 700, 377 (2002) [arXiv:nucl-th/0104030].

[24] S. R. Beane, P. F. Bedaque, T. C. Luu, K. Orginos, E. Pallante, A. Parreno and M. J. Savage [NPLQCD Collaboration], arXiv:hep-lat/0612026.

[25] I. Sato and P. F. Bedaque, arXiv:hep-lat/0702021.

[26] M. Luscher and P. Weisz, JHEP 0109, 010 (2001) [arXiv:hep-lat/0108014].

[27] P. F. Bedaque and A. Walker-Loud, Phys. Lett. B 660, 369 (2008) [arXiv:0708.0207 [hep-lat]]; P. F. Bedaque and A. Walker-Loud, arXiv:0811.2127 [hep-lat].

[28] K. R. Dienes, E. Dudas and T. Gherghetta, Nucl. Phys. B 537, 47 (1999) [arXiv:hep-ph/9806292]. 